\title{
Governo aberto e dados abertos governamentais: um mapeamento \\ e sistematização da produção acadêmica $^{1}$
}

OPEN GOVERNMENT AND OPEN GOVERNMENT DATA: A MAPPING AND SYSTEMATIZATION OF ACADEMIC PRODUCTION

\section{Maria Dominguez Costa Pinho ${ }^{2}$}

ORCID: https://orcid.org/0000-0003-2339-5570

(Universidade Federal da Bahia, Programa de Pós-Graduação em Comunicação e Cultura Contemporâneas. Salvador - BA, Brasil).

\section{Maria Paula Almada Silva}

ORCID: http://orcid.org/0000-0003-0037-614X

(Universidade Federal da Bahia, Programa de Pós-Graduação em Comunicação e Cultura Contemporâneas. Salvador - BA, Brasil). 


\section{Resumo}

Apesar de o crescimento da produção acadêmica sobre governo aberto e dados abertos governamentais, não há um trabalho que sistematize esta bibliografia, incluindo ambos temas. Esta pesquisa, então, propõe mapear e organizar sistematicamente a bibliografia sobre governo aberto e dados abertos governamentais. A amostra consta de $\mathrm{N}=150$ artigos, capítulos, livros, papers apresentados em congressos e relatórios em português e inglês. A pesquisa organiza os trabalhos encontrados considerando os seguintes metadados: autores e suas respectivas áreas do conhecimento, subtemas, padrão de investigação e ano de publicação. Os principais resultados mostram que a bibliografia está dividida em dez subtemas e é composta, principalmente, por artigos que tratam de dados abertos governamentais, usam métodos empíricos, e tratam de dois subtemas: "Implementação e Barreiras" e "Qualidade e Uso". Enfim, o trabalho sugere uma agenda de pesquisa para o futuro.

Palavras-chave: Revisão de literatura. Dados abertos governamentais. Governo aberto.

\section{Abstract}

Despite the growth of academic publications about open government and open government data, no systematic survey of this literature, including both topics, has been performed. This research then purposes to map and organize systematically the bibliography related to open government and open government data. The sample has $\mathrm{N}=150$ articles, chapters, books, papers presented in national and international congresses and reports in Portuguese and English. The research organizes the sample according to the following metadata: authors and their research areas, subthemes, inquiry patterns, and year of publication. The main results show that the bibliography is divided in ten subthemes and is made, mainly, of articles about open government data, apply empirical research, and refer to two subthemes: "Implementation and Barriers" and "Quality and Use". Finally, this work suggests future research agenda.

Keywords: Literature review. Open government data. Open government.

\section{Introdução}

Nos últimos anos, governos ao redor do mundo têm dedicado esforços para implementar programas de governo aberto e iniciativas de dados abertos governamentais para os mais diversos fins. A tendência é acompanhada pela produção acadêmica. Desde 2009, há quase uma década, tem-se notado um crescimento do volume de artigos, livros e relatórios publicados, além de papers apresentados em congressos nacionais e internacionais que tratam de governo aberto e dados abertos governamentais.

Desde então, entretanto, autores e pesquisadores das duas áreas não produziram uma revisão sistemática da literatura especializada. Este trabalho foi realizado, então, com a intenção de mapear a atenção acadêmica dada aos dois temas em questão com uma amostra de $\mathrm{N}=150$ trabalhos publicados. Foram encontradas revisões de literatura 
que tratam de subtemas específicos. É o caso de Attard e colegas (2015), que mapeiam iniciativas de dados abertos governamentais, focando em questões relacionados à publicação de dados, Charalabidis, Alexopoulos e Loukis (2016) propõem uma taxonomia das linhas de investigação encontradas na bibliografia sobre dados abertos governamentais, enquanto Hossain, Dwivedi e Rana (2014) realizam um estado da arte somente da produção acadêmica sobre dados abertos governamentais. Wirtz e Birkmeyer (2015), por sua vez, revisam a bibliografia de governo aberto, buscando uma definição conceitual mais precisa para o termo.

A proposta aqui é reunir em só uma bibliografia a produção acadêmica sobre governo aberto e dados abertos governamentais, já que ambos fazem parte dos mais recentes programas e iniciativas de governo digital. A hipótese defendida aqui é que como programas de governo aberto e iniciativas de dados abertos governamentais foram implementados e assumidos como tendência na gestão pública de maneira concomitante, sendo dados abertos governamentais considerados um dos principais impulsionadores do governo aberto (KUČERA; CHLAPEK; NEČASKÝ, 2013) as literaturas especializadas nos dois temas podem apresentar subtemas, autores e padrões de investigação similares.

Este trabalho está estruturado em três seções principais. Primeiro, o paper realiza uma breve revisão bibliográfica sobre governo aberto e dados abertos governamentais. Em seguida, expõe os procedimentos metodológicos utilizados por esta pesquisa e o último tópico, por sua vez, apresenta os resultados obtidos pela pesquisa.

\section{Sobre Governo aberto e Dados abertos governamentais}

Governo aberto surge como tendência em programas e ações governamentais ao redor do mundo a partir de 2009, com o início da gestão de Barack Obama como presidente dos Estados Unidos, quando foram estabelecidos três pilares fundamentais para as ações governamentais que seriam empreendidas ao longo do seu mandato: transparência, participação e colaboração. Depois do lançamento da política de Governo Aberto norteamericana, o programa de abertura do Reino Unido e o surgimento da Open Government Partnership (OGP) em 2011 foram decisivos para instalar-se uma tendência mundial para a adoção de programas de governo aberto em países de todos os continentes. A OGP é uma organização internacional que propõe estimular iniciativas de governo aberto pelo mundo, e atualmente conta com mais de 70 países associados ${ }^{4}$. Em 2011, a OGP foi

\footnotetext{
${ }^{4}$ A lista de países membros da OGP está disponível em: < http://www.opengovpartnership.org/countries $>$. Último acesso em: 01 jun. 2018.
} 
fundada por uma parceria entre 8 países, entre eles o Brasil, e desde então, a organização cria e estabelece planos de ação colaborativos com representantes de governos, ativistas, universidades e centros de pesquisa e organizações da sociedade civil, com a intenção implementar e acompanhar estes planos de ação e incitar práticas de governo aberto.

Apesar do termo contemporâneo datar de menos de uma década, desde a década de 60, pelo menos, as reivindicações por governos mais abertos cresceram, sobretudo após a implementação de leis de acesso à informação, e a pauta da abertura de dados e informações governamentais instalou-se internacionalmente. Em um primeiro momento, a ideia de governo aberto esteve relacionada à produção de transparência governamental $\mathrm{e}$ a expressão foi utilizada como sinônimo de governos que forneciam acesso a informações tradicionalmente restritas. Inclusive, a própria expressão parece ter sido utilizada pela primeira vez nos anos 50, justamente nos debates que precederam a aprovação da FOIA (freedom of information act) (YU; ROBINSON, 2012). Mesmo nos dias atuais, para alguns autores, a transparência continua sendo a característica mais importante (LORENZO; CÉSAR, 2010) e condição fundamental para as outras exigências do que se tem denominado por governo aberto. O uso contemporâneo do termo governo aberto, todavia, vai além do direito ao acesso a informações e não seria compreendido como é nos dias atuais sem o antecedente movimento software livre (LORENZO; CÉSAR, 2010), que cria as condições para práticas colaborativas na gestão governamental, como processos de co-criação e de crowdsourcing nos negócios públicos.

Para Lathrop e Ruma (2010), assim como o movimento open source permite que cidadãos contribuam com o código original de um software, o movimento pelo governo aberto implica que cidadãos não apenas tenham acesso a informações governamentais, como também possam colaborar com seus governos de maneira significativa. Nesse contexto, Yu e Robinson (2012) explicam que o vocábulo "aberto" detém duas conotações: tecnológica e filosófica. Enquanto a primeira remete ao uso de computadores para processar informações estruturadas e padronizadas, a segunda diz respeito à participação e à colaboração que devem ser parte fundamental das iniciativas de governo aberto. Esta "filosofia de governo do século XXI" (GÜEMES; RAMÍREZ-ALUJAS, 2012; OSZLAK, 2012) envolve, portanto, novos desafios à gestão pública, como o processamento e a publicação de um grande volume de dados e governamentais, a pressão pelo exercício do controle social e pela transparência pública.

Dados abertos governamentais, então, são considerados um dos principais impulsionadores do governo aberto (KUČERA; CHLAPEK; NEČASKÝ, 2013), e são 
apontados como capazes de fortalecer a transparência pública, a participação, fomentar a cocriação e a colaboração entre sociedade e governo. Nos últimos anos, este tipo de iniciativa tornou-se um dos principais elementos associados às reformas mais recentes na administração pública visando a promoção da transparência, o aperfeiçoamento na prestação de serviços públicos e outros fins (CLARKE; MARGETTS, 2014).

$\mathrm{O}$ movimento pelos dados abertos espalhou-se rapidamente com altas expectativas sobre os seus benefícios para a sociedade. Não só governos, mas empresas, universidades, bibliotecas e organizações multilaterais passaram a apostar na abertura de dados como meio de fortalecer a transparência, a confiança e o engajamento de clientes e usuários nos seus negócios. O crescimento das iniciativas de dados abertos caminha, assim, com a inovação e difusão de novos dispositivos digitais que possibilitam maior acessibilidade a dados (BRAUNSCHWEIG et al., 2012), além da facilidade de manuseio de ferramentas de processamento, armazenamento, manipulação, análise e distribuição deste vasto volume de dados (BOULTON; 2014; BOULTON et al., 2011).

$\mathrm{O}$ que difere dados abertos de outras informações governamentais quaisquer é o padrão do formato aberto, formulado por uma série de requisitos para que sejam acessados, reutilizados, lidos por máquinas e distribuídos livremente. Portanto, apesar das convergências, o movimento pelos dados abertos diverge das reivindicações prévias pois coloca ênfase em dados brutos em vez de informações interpretadas previamente, e uma formatação para que os dados sejam facilmente reutilizados e redistribuídos. Para Diniz (2010), informações governamentais são comumente publicadas em formatos que impedem que estas sejam acessíveis e utilizadas por todas as partes interessadas, como o formado PDF, impedindo, inclusive, que sejam combinadas e agregadas com outros dados.

Devido à rápida adoção aos dados abertos por governos do mundo todo, o tópico atraiu grande interesse da comunidade acadêmica, que, desde então, vem se dedicado a dar conta do fenômeno em suas diversas perspectivas. Janssen, Charalabidis e Zuiderwijk (2012), por exemplo, avaliam a política de dados abertos dos Países Baixos a partir de seis perspectivas: social e política, que diz respeito às implicações dos dados abertos no sistema político, na democracia e na vida dos cidadãos; econômica, que compreende o crescimento econômico, a geração de novos negócios e o estímulo à inovação; operacional e técnica, fazendo referência à habilidade para compreender e manusear os dados, à qualidade dos recursos e à disponibilização de dispositivos e ferramentas digitais; institucional, que avalia as políticas de dados abertos e a cultura de abertura da 
gestão pública; e, finalmente, a perspectiva legal, que demonstra a possibilidade de haver restrições legais para a publicação de dados.

A pesquisa sobre governo aberto e dados abertos governamentais conta com o risco de tomar como objeto um fenômeno recente e, por isso, evidências sobre os impactos econômicos, sociais e democráticos dos dados abertos, por exemplo, ainda são apontados como prematuros e pouco precisos pela bibliografia. Apesar disto, a literatura no tema se posiciona, em sua maioria, com grande otimismo para a adoção de programas de governo aberto e iniciativas de dados abertos governamentais e aponta para efeitos positivos que estas iniciativas teriam na governança democrática.

A este ponto, no qual governos dos três níveis ao redor do mundo parecem terem aderido à tendência de programas de governo aberto e iniciativas de dados abertos governamentais, e após quase uma década do início da gestão de Barack Obama como presidente dos Estados Unidos, que marcou o início da referida tendência, temos condições de reunir a produção acadêmica sobre os temas e a formação desta nova área de estudos, seguindo Gomes (2016), que fiz que a atenção acadêmica é sempre um sinal da atenção e das preocupações de toda a sociedade em um tema ou assunto.

\section{Procedimentos metodológicos}

Uma vez definido que este estudo se dedicaria a coletar e sistematizar a produção acadêmica referente aos dois temas (governo aberto e dados abertos governamentais), a pesquisa buscou títulos de livros, artigos, papers apresentados em congressos nacionais e internacionais e relatórios de organizações multilaterais. Para isso, buscou-se pelas expressões "governo aberto", "dados abertos governamentais", "open government" e "open government data" em revistas científicas das áreas de Ciências Sociais, Administração Pública, Ciência Política e Comunicação, em sites de repositórios de artigos científicos (Portal de periódicos Capes, Google Scholar, Research Gate), além de artigos coletados do arquivo pessoal da pesquisadora. Além desta primeira busca geral, buscou-se pelas expressões em portais específicos de eventos, organizações e revistas científicas que tratam do tema, tais quais: o evento internacional Global Conference on Transparency Research; as organizações internacionais Open Government Partnership (OGP), Open Knowledge Foundation e Organisation for Economic Co-operation and Development (OECD), além das revistas Government Information Quarterly Journal, Transforming Government: People, Process and Policy, Electronic Journal of e-Government, Social 
Science Computer Review, International Journal of Public Administration, Public Administration, e a Policy \& Internet.

Enfim, chegou-se a um corpus de 150 títulos, entre eles artigos científicos, papers apresentados em anais de congressos, livros e relatórios coletados. Só foram selecionados para este trabalho os trabalhos que contém "governo aberto", "dados abertos governamentais", "open government" ou "open government data" no título ou no resumo dos trabalhos. Os metadados referentes à amostra foram organizados em uma planilha no Google Sheets e na plataforma Mendeley. Em seguida, todos os resumos, palavras-chave e boa parte dos artigos completos foram lidos.

A bibliografia foi sistematizada de acordo com os seguintes critérios:

1. Título: título do documento;

2. Autor(es): nome dos autores e das suas respectivas universidades ou instituições de trabalho;

3. Ano: identificação do ano de publicação ou apresentação do trabalho;

4. Área de formação dos autores: identificação da área de formação dos autores entre Administração, Comunicação, Ciência Política, Direito ou Ciência da Computação e Informação;

5. Área geral: distinção entre trabalhos que tratam de Governo Aberto ou de Dados Abertos Governamentais ou ambos;

6. Padrão de investigação: distinção entre trabalho empírico ou teórico;

7. Tipo de publicação: identificação do documento entre artigo, paper apresentado em congresso, livro ou relatório;

8. Subtemas: os títulos foram categorizados com um ou mais subtemas aos quais se referem, sendo eles:

Tabela 1 - Divisão da bibliografia em dez subtemas

\begin{tabular}{|c|c|c|}
\hline Subtemas & Descrição & Exemplos \\
Transparência, & $\begin{array}{c}\text { Trabalhos que verificam graus de } \\
\text { transparência produzidos por programas de } \\
\text { governo aberto e portais de dados abertos, } \\
\text { Acestability e Leis de à Informação } \\
\text { avaliam leis de acesso à informação, } \\
\text { iniciativas criadas com o objetivo de } \\
\text { produzir mais accountability, etc. }\end{array}$ & $\begin{array}{c}\text { Murillo (2013) } \\
\text { Macadar, Klein, Luciano } \\
\text { Lourenço (2015) } \\
\text { Corrêa et al (2017) } \\
\text { Janssen et al (2017) }\end{array}$ \\
\hline
\end{tabular}




\begin{tabular}{|c|c|c|}
\hline Qualidade e Uso & $\begin{array}{l}\text { Avaliam a qualidade de dados } \\
\text { publicados em portais de dados abertos } \\
\text { governamentais a partir de critérios como } \\
\text { usabilidade, atualidade, formatação, etc. } \\
\text { Aqui, são utilizados índices de dados } \\
\text { abertos e critérios estabelecidos para aferir } \\
\text { a qualidade mínima para que dados sejam } \\
\text { considerados abertos. Além disso, muitos } \\
\text { trabalhos propõem mapear os usos que } \\
\text { se faz dos dados, para quais finalidades e } \\
\text { quais são as condições necessárias para o } \\
\text { uso efetivo dos dados abertos. }\end{array}$ & $\begin{array}{c}\text { O’Hara (2012) } \\
\text { Kučera, Chlapek, Nečaský } \\
\text { (2013) } \\
\text { Bright et al (2015) } \\
\text { Saéz Martin, De Rosario, } \\
\text { Perez (2016) } \\
\text { Zuiderwijk, Janssen, Dwivedi } \\
\text { (2015) }\end{array}$ \\
\hline $\begin{array}{c}\text { Intermediários e Partes } \\
\text { interessadas }\end{array}$ & $\begin{array}{l}\text { Alguns trabalhos sugerem avaliar o papel } \\
\text { dos intermediários ou partes interessadas } \\
\text { (stakeholders) em iniciativas de dados } \\
\text { abertos governamentais. Aqui, estes } \\
\text { são considerados como pontes entre os } \\
\text { produtores dos dados (governos) e os } \\
\text { cidadãos, ao traduzir e transformar os } \\
\text { dados em iniciativas, projetos e aplicativos, } \\
\text { por exemplo. Normalmente, ativistas dos } \\
\text { dados abertos, jornalistas, programadores, } \\
\text { organizações sem fins lucrativos e gestores } \\
\text { públicos são apontados como principais } \\
\text { partes interessadas. }\end{array}$ & $\begin{array}{c}\text { Gonzalez-Zapata, Heeks } \\
\text { (2015) } \\
\text { Chan, Johnson, Shookner } \\
\text { (2016) } \\
\text { Schrock (2016) } \\
\text { Schrock, Shaffer (2017) } \\
\text { Craveiro, Albano (2017) }\end{array}$ \\
\hline $\begin{array}{c}\text { Implementação e } \\
\text { Barreiras }\end{array}$ & $\begin{array}{c}\text { Parte da literatura se dedica a mapear } \\
\text { e propor soluções para as principais } \\
\text { barreiras à implementação de programas } \\
\text { de governo aberto e portais de dados } \\
\text { abertos. Entre as principais barreiras, } \\
\text { estão as limitações nos recursos humanos } \\
\text { e financeiros, falta de vontade política, e } \\
\text { dificuldades operacionais e tecnológicas da } \\
\text { administração pública. }\end{array}$ & $\begin{array}{l}\text { Janssen, Charalabidis, } \\
\text { Zuiderwijk (2012) } \\
\text { Zuiderwijk et al (2014) } \\
\text { Yang, Lo, Shiang (2015) } \\
\text { Wang, Lo (2015) } \\
\text { Yang, Wu (2016) }\end{array}$ \\
\hline $\begin{array}{l}\text { Política Pública e } \\
\text { Iniciativas }\end{array}$ & $\begin{array}{c}\text { Alguns autores investigam políticas } \\
\text { públicas, programas governamentais, ações } \\
\text { ou diretrizes de governo aberto e iniciativas } \\
\text { de dados abertos a partir de análises } \\
\text { documentais, por exemplo. Na maioria dos } \\
\text { casos encontrados, a política de algum país, } \\
\text { estado ou cidade é tomado como objeto de } \\
\text { estudo. }\end{array}$ & $\begin{array}{c}\text { Coglianese (2009) } \\
\text { Bates (2014) } \\
\text { OECD (2014) } \\
\text { Attard et al (2015) } \\
\text { De Blasio, Selva (2016) }\end{array}$ \\
\hline Participação & $\begin{array}{l}\text { Aqui, os trabalhos encontrados investigam } \\
\text { se programas de governo aberto e } \\
\text { iniciativas de dados abertos governamentais } \\
\text { aumentam a participação dos cidadãos nos } \\
\text { negócios públicos. Além disso, se e como } \\
\text { estes programas e iniciativas investem em } \\
\text { métodos de co-criação, crowdsourcing, etc. }\end{array}$ & $\begin{array}{c}\text { Andrews, Corrêa (2013) } \\
\text { Wijnhoven, Ehrenhard, Kuhn } \\
\text { (2015) } \\
\text { Piscopo, Siebes, Hardman } \\
\text { (2017) } \\
\text { Hivon, Titah (2017) } \\
\text { Wirtz, Weyerer, Rösch (2017) }\end{array}$ \\
\hline
\end{tabular}




\begin{tabular}{|c|c|c|}
\hline $\begin{array}{c}\text { Teoria de governo } \\
\text { e dados abertos } \\
\text { governamentais }\end{array}$ & $\begin{array}{c}\text { Programas de governo aberto e iniciativas } \\
\text { de dados abertos governamentais são } \\
\text { novos e não há teorias consistentes para os } \\
\text { fenômenos. Sendo assim, alguns trabalhos } \\
\text { se dedicam a encontrar marcos teóricos } \\
\text { comuns para os temas, além de levantar } \\
\text { suas questões, dimensões e problemas de } \\
\text { pesquisa mais recorrentes nas bibliografias. }\end{array}$ & $\begin{array}{c}\text { Francoli (2011) } \\
\text { Corojan, Domínguez (2011) } \\
\text { Meijer, Curtin, Hillebrandt } \\
\text { (2012) } \\
\text { Pyrozhenko (2017) } \\
\text { Wirtz, Birkmeyer (2015) }\end{array}$ \\
\hline Outros & $\begin{array}{c}\text { Além das sete categorias descritas acima, } \\
\text { foram encontrados trabalhos sobre } \\
\text { governos e dados abertos que enfatizam em } \\
\text { teoria democrática; valor econômico dos } \\
\text { dados abertos; redes sociais digitais; dados } \\
\text { abertos linkados e ecossistemas de governo } \\
\text { aberto. }\end{array}$ & $\begin{array}{c}\text { Hendler et al (2011) } \\
\text { Geiger, Von Lucke (2012) } \\
\text { Lee, Kwak (2012) } \\
\text { Harisson, Pardo, Cook (2012) } \\
\text { Iglesia (2013) }\end{array}$ \\
\hline
\end{tabular}

Fonte: Elaboração própria

\section{Resultados}

A análise da bibliografia foi realizada de acordo com os tópicos mencionados na seção anterior deste artigo. Aqui, a organização, sistematização e cruzamento dos metadados da amostra de $\mathrm{N}=150$ documentos será apresento nos seguintes gráficos e tabelas.

\section{Resultados das categorias de análise}

\section{Área de formação dos autores}

A bibliografia sobre governo e dados abertos governamentais une fenômenos que podem ser abordados por diversas perspectivas (política, social, econômica, técnica e operacional, entre outras). Por isso, a produção acadêmica sobre governo aberto e dados abertos governamentais mostrou ser também multidisciplinar. Nesta etapa da análise, buscou-se a área de formação e/ou atuação de trabalho e estudos dos autores responsáveis pelos artigos, relatórios, livros e papers coletados. Estes dados foram identificados com uma busca pelo currículo ou website pessoal dos autores de cada documento. Em caso de identificação de um artigo com diversos autores, por exemplo, foi contabilizada a área de formação do primeiro autor ou autora da publicação. Enfim, foram identificadas dez áreas, sendo Administração a mais proeminente, com 67 autores encontrados, o que equivale a, em média, a 44,6\% da amostra. 
Gráfico 1 - Áreas de formação dos autores

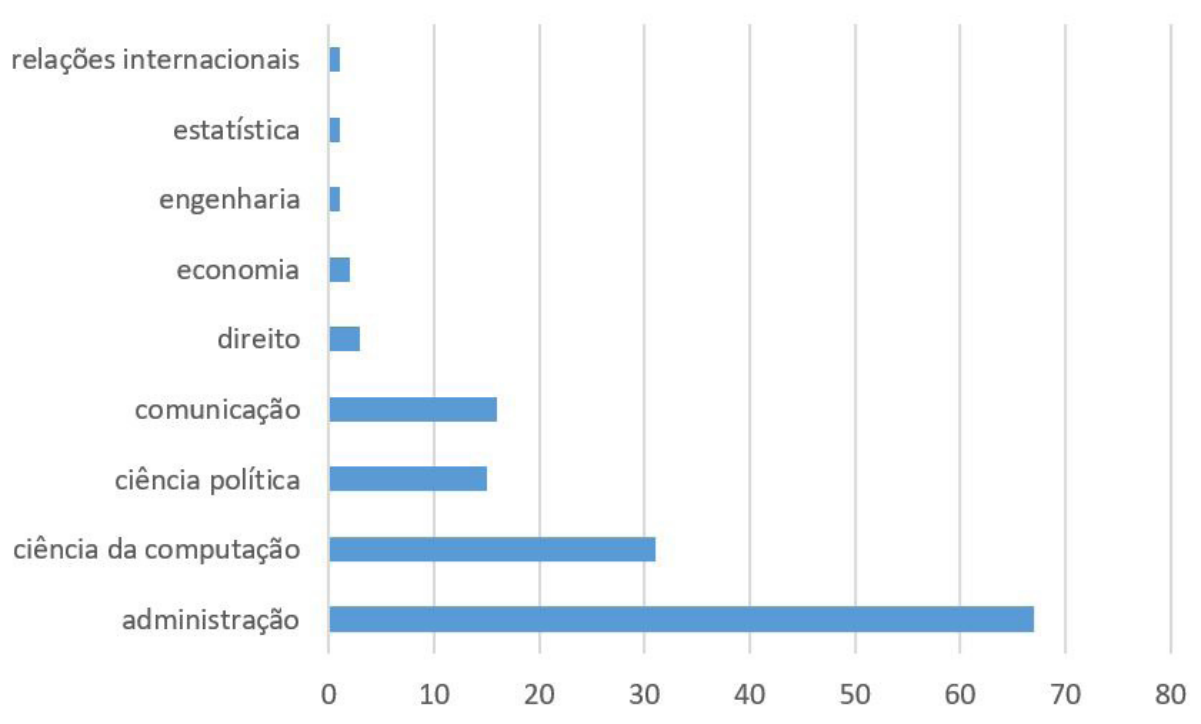

Fonte: Elaboração própria

\section{Ano de publicação ou apresentação}

Uma questão possível de ser respondida com a sistematização e análise da bibliografia é: quando foram inaugurados os estudos sobre governo aberto e dados abertos governamentais? Apesar do tema do "governo aberto" não ser novo, antes de 2009 ele foi associado à ideia de transparência governamental e às discussões sobre leis de acesso à informação. Nesse sentido, um governo aberto era o mesmo que publicava informações que antes eram consideradas como confidenciais. $\mathrm{O}$ uso contemporâneo do termo, por outro lado, amplia o seu sentido e, sobretudo, seguindo da Declaração pelo Governo Aberto de Barack Obama, publicada no primeiro dia de sua gestão como presidente dos Estados Unidos, em 2009, passa a ser definido como governo aberto aquele que produz transparência, participação e colaboração.

Para incluir na amostra desta pesquisa apenas aqueles trabalhos que utilizam a conotação contemporânea do termo governo aberto, foram considerados apenas aqueles publicados após os anos 2000. Vimos que apenas dois trabalhos (um artigo e dois relatórios) foram publicados antes de 2009, e enquanto um se referia à transparência e lei de acesso à informação (CHANCE; LOCKE, 2007), o outro investigou boas práticas de transparência e participação (OECD, 2003). Tomando como referência o ano de 2009, o primeiro artigo encontrado na amostra trata justamente da política de governo aberto de Obama (COGLIANESE, 2009), entendida neste trabalho como um marco para a 
bibliografia. O Gráfico 2, abaixo, mostra que, de fato, a partir de 2010, há um incremento constante na produção acadêmica nas referidas áreas de pesquisa, com uma queda em 2013, e mais um crescimento em 2014, até a produção chegar ao até então auge na atenção acadêmica com uma produção de 30 publicações no referido ano, mesma quantidade de títulos publicados ou apresentados em 2017.

Gráfico 2 - Distribuição cronológica da produção acadêmica (2003 - 2017)

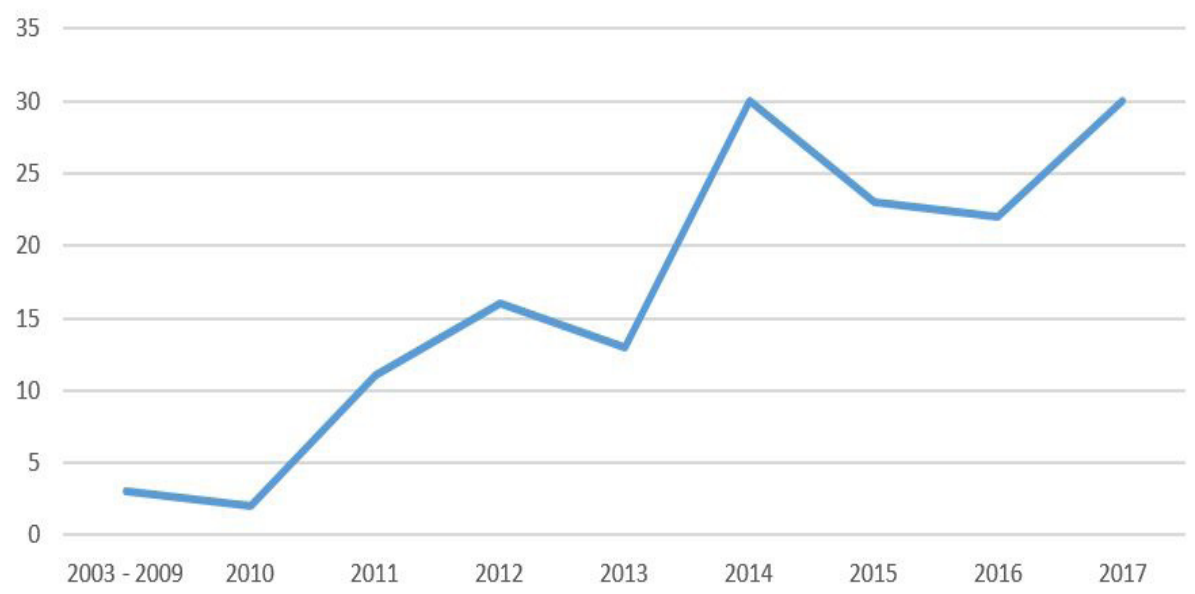

Fonte: Elaboração própria

\section{Temas gerais}

Entre os dois temas considerados nesta pesquisa, dados abertos governamentais recebeu maior atenção acadêmica, chegando próximo a dois terços da amostra $(\mathrm{N}=$ 97), ou em média, $65 \%$ dos trabalhos coletados. O tema do governo aberto, por sua vez, contabilizou um total de 53 trabalhos publicados no período considerado na amostra, como mostra o gráfico abaixo. 
Gráfico 3 - Temas gerais dos artigos: governo aberto ou dados abertos governamentais

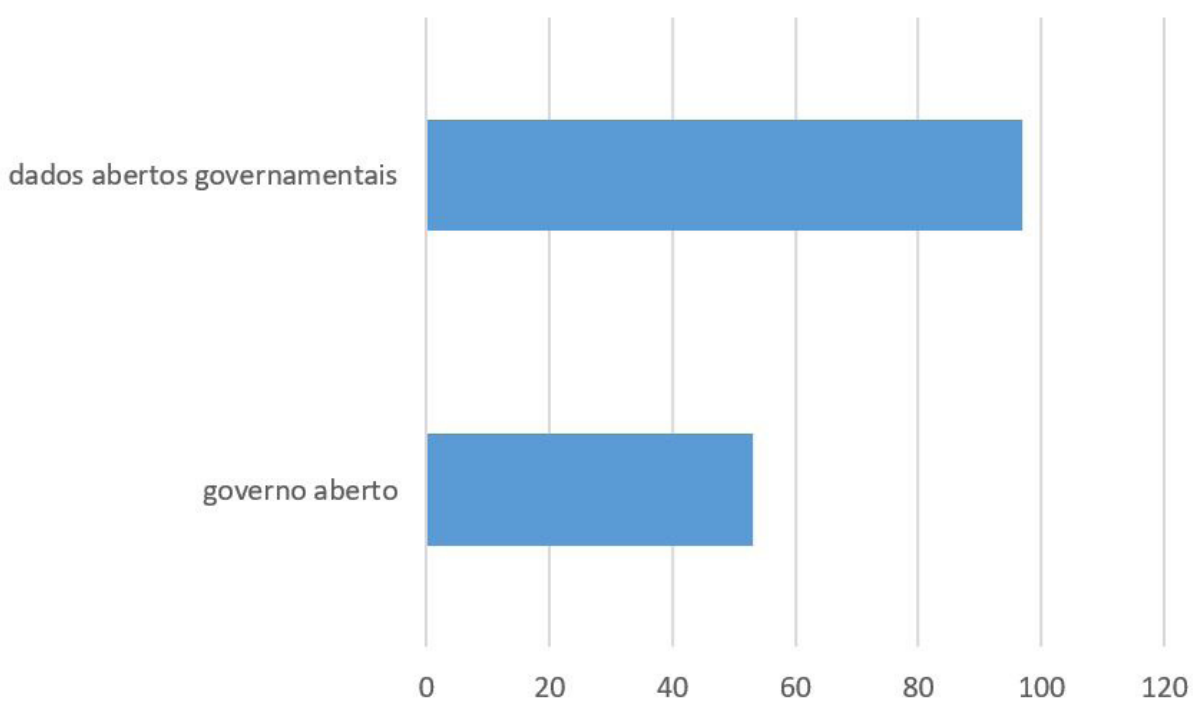

Fonte: Elaboração própria

\section{Tipo de publicação}

O Gráfico 4 demonstra os tipos de publicações encontrados na amostra entre artigos em revistas científicas, papers em congressos, relatórios e livros. A maior parte da bibliografia é formada por artigos, equivalente a 76,6\% da amostra, seguidos de 12,6\% de relatórios, 9,3\% de papers apresentados em congressos nacionais e internacionais e, finalmente, apenas aproximadamente 1,5\% da amostra composta por livros.

Entre os artigos, a revista científica que recebeu o maior volume de publicações sobre os temas foi a Government Information Quarterly, seguida da Transforming Government: People, Process and Policy. Os relatórios, por sua vez, foram produzidos e publicados, principalmente, por organizações multilaterais como a OECD, World Wide Web Foundation, The Open Data Institute, Open Knowledge Foundation e Open Government Partnership. 
Gráfico 4 - Tipo de trabalho publicado

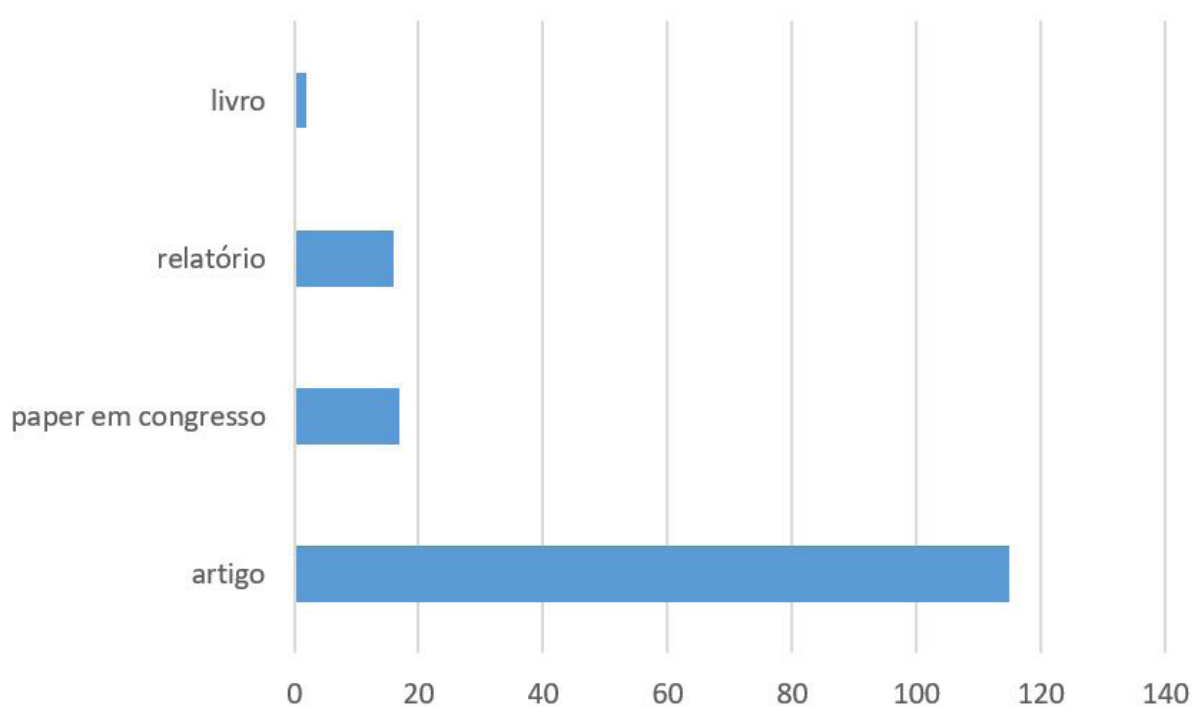

Fonte: Elaboração própria

\section{Análise comparativa dos resultados}

\section{Subtemas $\times$ Ano}

Que subtemas tratados na bibliografia receberam maior atenção acadêmica ao longo do período avaliado? Podemos responder esta pergunta com a avaliação do Gráfico 5, abaixo, que esclarece a distribuição cronológica da publicação por subtemas. O Gráfico elucida, ainda, a geometria encontrada no Gráfico 2, que mostrou um maior número de publicações e apresentações nos anos de 2014 e 2017. Abaixo, vemos que, na verdade, enquanto o ritmo de publicações se manteve constante durante quase todo período avaliado (com picos em 2012, 2014 e 2017), a atenção acadêmica voltada para o subtema "Implementação e Barreiras" acompanhou os picos de crescimento da literatura em 2012 e 2014, enquanto a produção foi mais distribuída em subtemas em 2017. Outro tema que colabora para o crescimento da literatura em 2014 é o "Políticas Públicas e Iniciativas", que também demonstra um pico no mesmo período. 
Gráfico 5 - Distribuição cronológica da publicação dos subtemas específicos

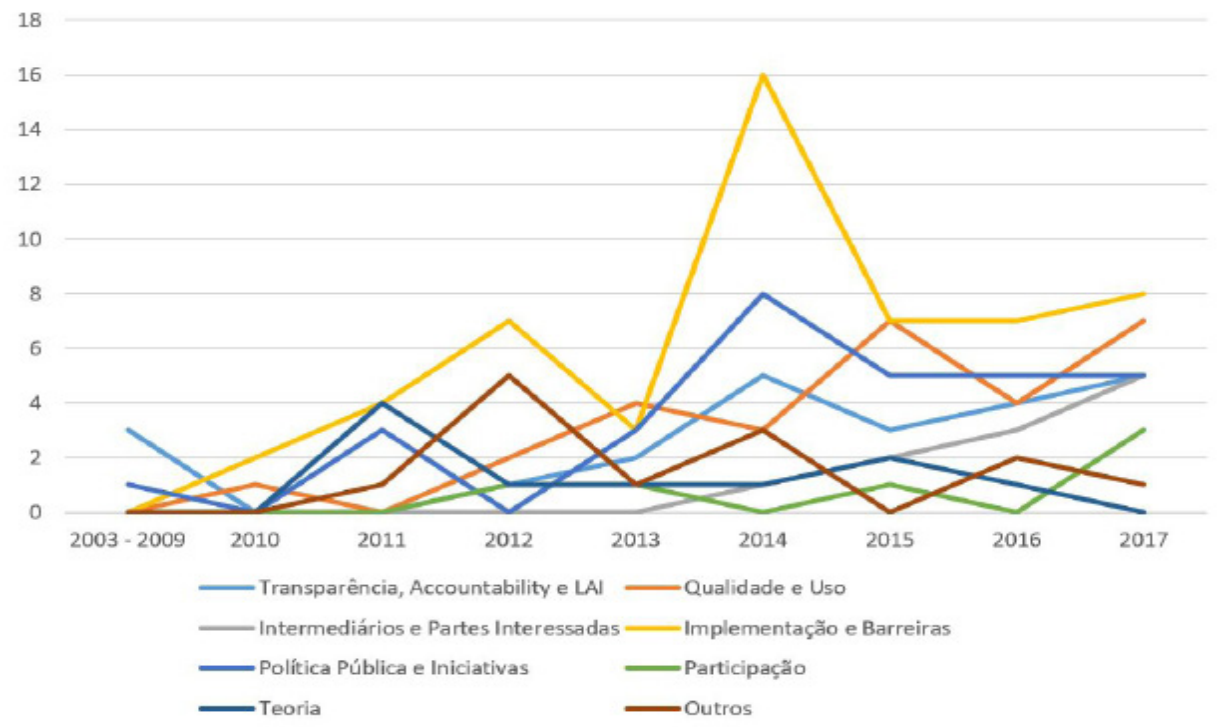

Fonte: Elaboração própria

\section{Tema geral x Subtemas}

Apesar de similares, os temas governo aberto e dados abertos governamentais correspondem a fenômenos distintos e, por isso, apresentam seus próprios problemas e questões de pesquisa. Os Gráficos 6 e 7 mostram a proporção da atenção acadêmica dada a cada subtema, separadamente, para dados abertos governamentais e governo aberto, respectivamente.

Como similaridade, vemos que autores e pesquisas de ambas as áreas publicaram, principalmente, sobre "Implementação e Barreiras". O Gráfico 6 mostra que além deste, o segundo subtema proeminente nos estudos sobre dados abertos governamentais é o de Qualidade e Uso dos dados. O Gráfico 7 mostra que, por outro lado, a pesquisa sobre governo aberto dedica atenção principalmente ao tema da Implementação e Barreiras, seguido do tema Política Pública e Iniciativas, e a terceira categoria é a sobre Transparência e Accountability. De fato, tal resultado pode decorrer da rápida adesão a programas e políticas de governo aberto por governos municipais, estaduais e federais ao redor do mundo, desde 2009. O resultado pode demonstrar um interesse da literatura em avaliar e acompanhar essas iniciativas. Além disso, como já vimos, o tema do governo aberto sempre esteve relacionado à transparência, e este continua sendo considerado o principal elemento do governo aberto para alguns autores. 
Gráfico 6 - Distribuição de temas específicos na bibliografia sobre dados abertos governamentais

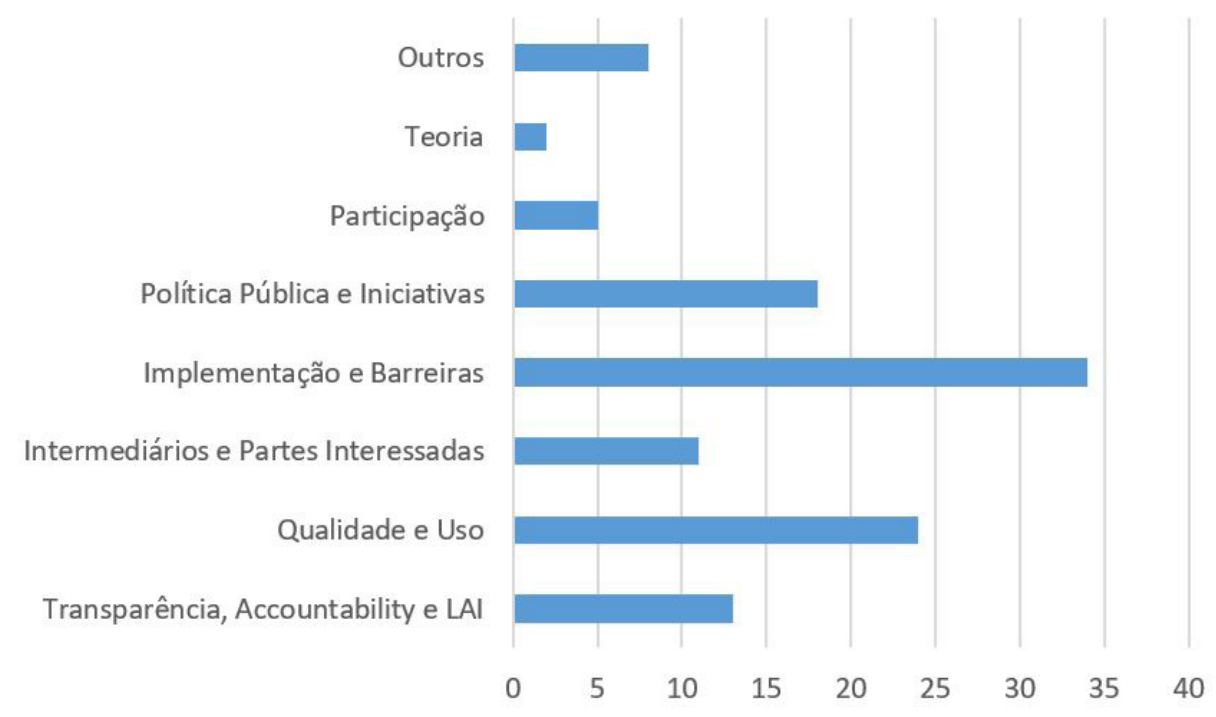

Fonte: Elaboração própria

Gráfico 7 - Distribuição de temas específicos na bibliografia sobre governo aberto

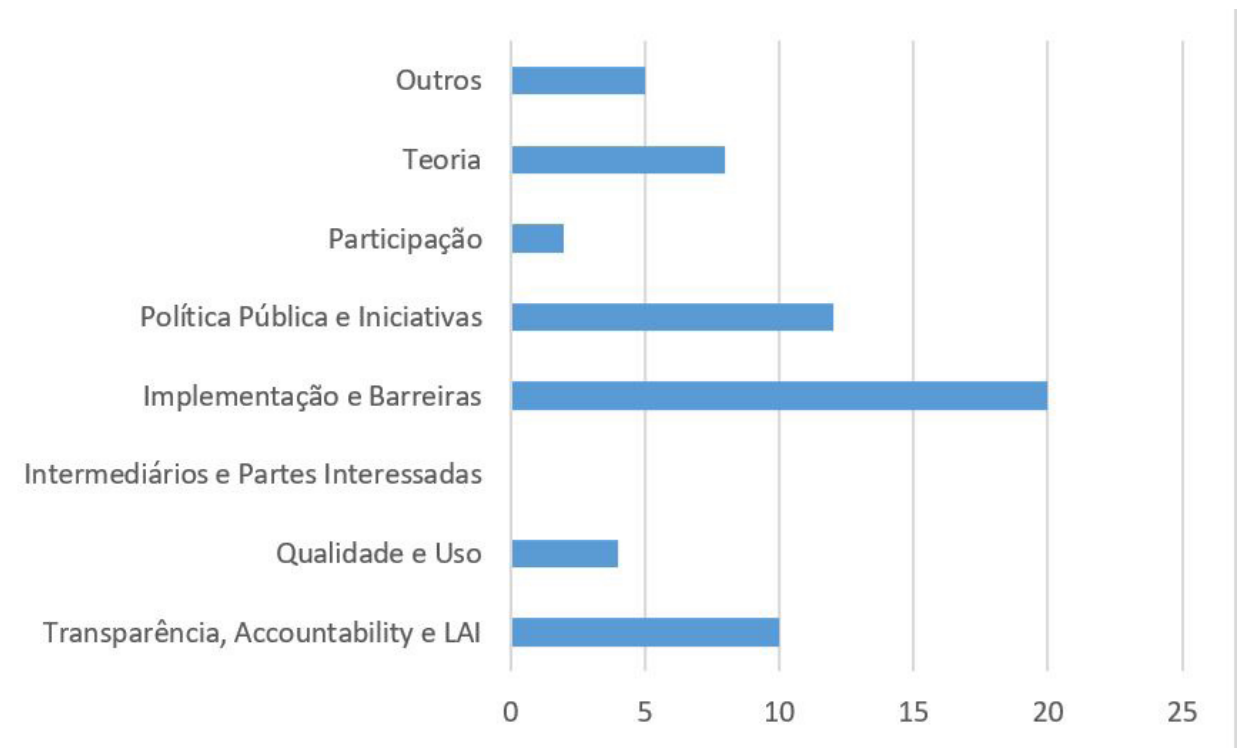

Fonte: Elaboração própria

\section{Subtemas X Padrão de investigação}

O padrão de investigação proeminente entre os trabalhos encontrados foi $\mathrm{o}$ empírico, com 107 dos 150 trabalhos encontrados, ou aproximadamente $71 \%$ da amostra, contra 43 ou uma porcentagem de aproximadamente $28 \%$ de trabalhos predominantemente 
teóricos. Este resultado pode ser devido aos fenômenos estudados serem recentes, com programas de governo aberto e iniciativas de dados abertos governamentais datando de, no máximo, uma década. Desse modo, as literaturas especializadas podem contar com um relevante número de ações e iniciativas para serem avaliadas, mas com um referencial teórico ainda em construção.

Além disso, estes resultados devem-se ao tema "governo aberto" ainda ser considerado um conceito em construção e, por isso, é difícil apontar uma definição única e consensual que o delimite precisamente (COBO, 2012; MARISCAL, 2012). Desse modo, talvez seja mais proveitoso compreendê-lo a partir dos princípios que evoca e das iniciativas que empreende por meio de estudos de caso e avaliação de políticas públicas, portais de dados abertos e programas de governo aberto, por exemplo, empregando procedimentos metodológicos do tipo empírico.

Vemos no Gráfico 8 que os trabalhos empíricos dedicam-se, sobretudo, às categorias que tratam de "Implementação e Barreiras", "Qualidade e Uso", "Política Pública e Iniciativas" e a "Intermediários e Partes Interessadas", esta última, que recebe atenção inteiramente de trabalhos empíricos. Os trabalhos teóricos, por sua vez, tratam de "Transparência, Accountability e LAI", e, evidentemente, sobre a "Teoria" de governo aberto.

Gráfico 8 - Padrões de investigação utilizados nos trabalhos

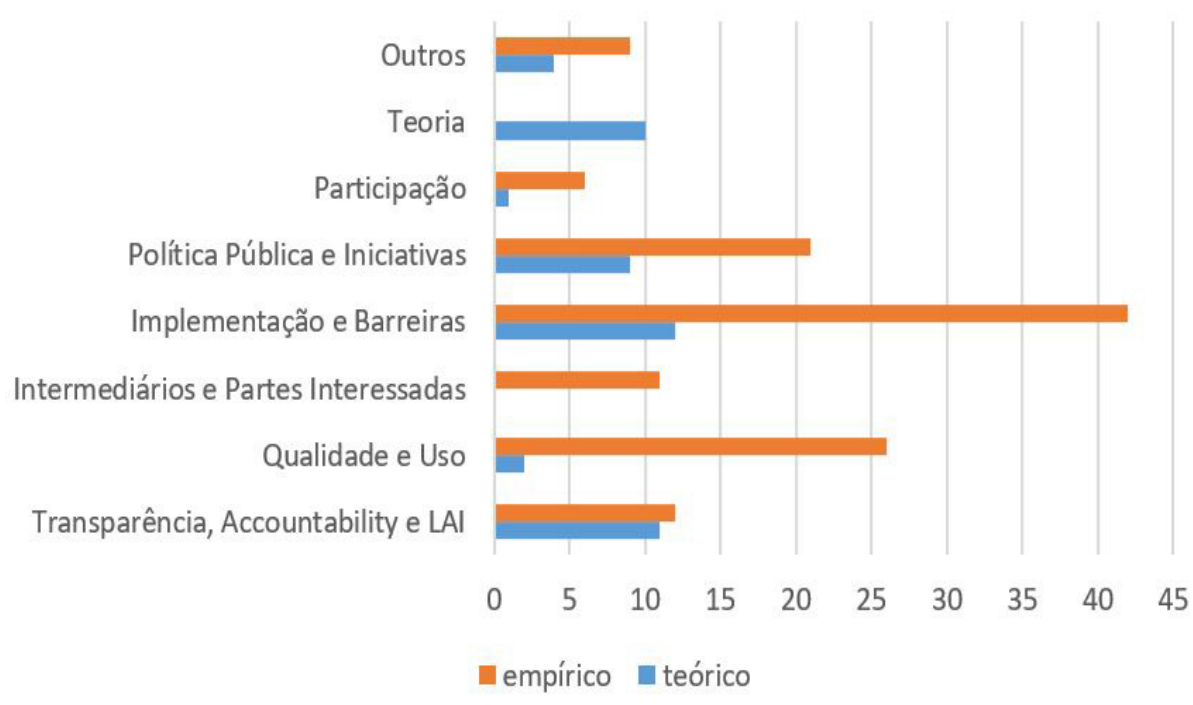

Fonte: Elaboração própria 


\section{Considerações finais}

A amostra encontrada neste estudo mostra um constante interesse da comunidade acadêmica para os temas do governo aberto e dados abertos governamentais. Se a bibliografia, por um lado, data de pouco menos de uma década, por outro, mostra uma variedade de subtemas aos quais se dedicada ao longo do período investigado neste trabalho. A amostra é composta por 150 títulos de artigos, livros, papers apresentados em congressos e relatórios nacionais e internacionais.

Apesar do campo de pesquisa ser recente e estar em período de formação, sobretudo quando comparado a outras áreas de pesquisa consolidadas que tratam de governos e política em ambientes digitais, a análise da bibliografia em questão mostrou um interesse e dedicação constantes da atenção acadêmica ao longo da última década. Foram encontrados oito subtemas - Transparência, Accountability e LAI, Participação, Implementação e Barreiras, Intermediários e Partes Interessadas, Políticas Públicas e Iniciativas, Qualidade e Uso dos Dados, Teoria de Governo Aberto e Dados Abertos Governamentais e Outros - que representam dimensões, temas, questões de pesquisa e enfoques distintos. Um resultado desta pesquisa foi que os subtemas Implementação e Barreiras, Qualidade e Uso e Política Pública e Iniciativas foram os proeminentes na amostra global. A maior atenção dada a estes temas pode ser decorrente de outro resultado deste estudo: o fato de que a maioria dos estudiosos sobre governos e dados abertos advêm da área da Administração Pública. Sendo assim, se interessam pelos processos, barreiras e meios de implementar programas e políticas públicas como estes, bem como pelo resultado destas políticas e programas, compreendido aqui como o uso social desses dados. Une-se a isso o caráter mais empírico do que teórico dos títulos encontrados, demonstrando que a bibliografia encontra-se no estágio de discutir e avaliar iniciativas de governo aberto e de dados abertos governamentais, mais que formar uma teoria propriamente dita para estes fenômenos.

Mais pesquisa é necessária para identificar os principais problemas de pesquisa encontrados nesta bibliografia, além dos procedimentos metodológicos mais recorrentes, realizando uma subdivisão, por exemplo, entre os casos que aplicam métodos como estudos de caso, entrevistas, análises comparativas, e outros. Desde já, todavia, é possível indicar que novos trabalhos tratem teoricamente dos fenômenos encontrados ou, que, pelo menos, o associem a outras teorias e linhas de pesquisa já existentes que tratam de iniciativas digitais e suas implicações para a democracia, ainda que se tratem 
de fenômenos distintos, como é o caso das pesquisas sobre governo digital (digital ou e-government) e democracia digital (digital ou e-democracy). Assim, espera-se que, junto a este trabalho, um mapeamento ainda mais específico da bibliografia sobre governo aberto e dados abertos governamentais possa colaborar com o futuro da pesquisa nos dois temas em questão, oferecendo novos insights para perguntas de pesquisa teóricas, questões empíricas, e possíveis novos subtemas.

\section{Referências}

ANDREWS, P; CORRÊA, F. Using parliamentary open data to improve participation. In: INTERNATIONAL CONFERENCE ON THEORY AND PRACTICE OF ELECTRONIC GOVERNANCE. 7., 2013. Proceedings [...]. ACM, 2013. p. 242-249.

ATTARD, J.; et al. A systematic review of open government data initiatives. Government Information Quarterly. Elsevier Inc. v. 32, n. 4, p. 399-418, 2015.

BATES, J. The strategic importance of information policy for the contemporary neoliberal state: The case of Open Government Data in the United Kingdom. Government Information Quarterly, v. 31, n. 3, p. 388-395, 2014.

BOULTON, G. The open data imperative, Insights, v. 27, n. 2, p. 133-138, 2014.

BOULTON, G. et al. Science as a public enterprise: The case for open data. The Lancet, v. 377, n. 9778 , p. 1633-1635, 2011.

BRAUNSCHWEIG, K. et al. The State of Open Data. Limits of Current Open Data Platforms. Paper read at International World Wide Web Conference, Lyon, France, 2012.

BRIGHT, J., et al. Explaining usage patterns in Open Government Data: the case of Data.gov. uk. Oxford Internet Institute [online], pp. 1-22, 2015. Disponível em: http://www.icpublicpolicy. org/conference/file/reponse/1433323028.pdf. Acesso em: 15 mar. 2018.

CHAN, M.; JOHNSON, P.; SHOOKNER, M.. Assessing the Use of Government Open Data and the Role of Data Infomediaries The Case of Nova Scotia's Community Counts Program. JeDEMeJournal of eDemocracy and Open Government, v. 8, n. 1, p. 1-27, 2016. 
CHARALABIDIS, Y.; ALEXOPOULOS, C.; LOUKIS, E. A taxonomy of open government data research areas and topics. Journal of Organizational Computing and Electronic Commerce, v. 26, n. 1-2, p. 41-63, 2016.

CLARKE, A.; MARGETTS, H. Governments and Citizens Getting to Know Each Other? Open, Closed, and Big Data in Public Management Reform. Policy \& Internet, v. 6, n. 4, p. 393-417, 2014.

COBO, C. Gobierno abierto: de la transparencia a la inteligencia cívica. In: HOFMANN, A.; RAMÍREZ-ALUJAS, A. V.; PEREZNIETO, J. A. B. (Org.). La promesa del gobierno abierto. México, DF: Instituto de Acceso a la Información Pública y Protección de Datos Personales del Distrito Federal, 2012.

COGLIANESE, C. The transparency president? The Obama administration and open government. Governance, v. 22, n. 4, p. 529-544, 2009.

CRAVEIRO, G.; ALBANO, C. Open data intermediaries: coproduction in budget transparency. Transforming Government: People, Process and Policy, v. 11, n. 1, p. 119-131, 2017.

CORRÊA, A. et al. Transparency and open government data: a wide national assessment of data openness in Brazilian local governments. Transforming Government: People, Process and Policy, v. 11, n. 1, p. 58-78, 2017.

DE BLASIO, E.; SELVA, D. Why choose open government? motivations for the adoption of open government policies in four european countries. Policy \& Internet, v. 8, n. 3, p. 225-247, 2016.

DINIZ, V. Como conseguir dados governamentais abertos. In: CONGRESSO CONSAD DE GESTÃo PÚBLICA, 3., 2010, Brasília, DF. Anais Eletrônicos [...]. Brasília: CONSAD, 2010. Disponível em: https://i3gov.planejamento.gov.br/como_conseguir_dados_governamentais_ abertos.pdf. Acesso em: 15 mar. 2018.

FRANCOLI, M. What Makes Governments' Open'? Sketching out Models of Open Government. eJournal of eDecomcracy \& Open Government, v. 3, n. 2, p. 152-165. 2011.

GOMES, W. 20 Anos de Política, Estado e Democracia Digitais: Uma cartografia do campo. In: SILVA, Sivaldo P.; BRAGATTO, Rachel C.; SAMPAIO, R. C.. (Org.). Democracia digital, comunicação política e redes: teoria e prática. São Paulo: Letra \& Imagem: Letra \& Imagem, 2016, p. 25-45. 
GONZALEZ-ZAPATA, F.; HEEKS, R. The multiple meanings of open government data: Understanding different stakeholders and their perspectives. Government Information Quarterly, v. 32, n. 4, p. 441-452, 2015.

GÜEMES, M. C.; RAMÍREZ-ALUJAS, A. V. Gobierno abierto, reforma del Estado y modernización de la gestión pública: alcances, obstáculos y perspectivas en clave Latinoamericana. In: HOFMANN, A.; RAMÍREZ-ALUJAS, A. V.; PEREZNIETO, J. A. B. (Org.). La promesa del gobierno abierto. México, DF: Instituto de Acceso a la Información Pública y Protección de Datos Personales del Distrito Federal, 2012.

HENDLER, J. et al. US government linked open data: semantic. data. gov. IEEE Intelligent Systems, v. 27, n. 3, p. 25-31, 2012.

HIVON, J.; TITAH, R. Conceptualizing citizen participation in open data use at the city level. Transforming Government: People, Process and Policy, v. 11, n. 1, p. 99-118, 2017.

HOSSAIN, M. A.; DWIVEDI, Y. K.; RANA, N.P. State-of-the-art in open data research: Insights from existing literature and a research agenda. Journal of Organizational Computing and Electronic Commerce, v. 26, n. 1-2, p. 14-40. 2016.

HUIJBOOM, N.; VAN DEN BROEK, T. Open data: an international comparison of strategies. European journal of ePractice, v. 12, n. 1, p. 4-16, 2011.

JANSSEN, M.; CHARALABIDIS, Y.; ZUIDERWIJK, A. Benefits, adoption barriers and myths of open data and open government. Information Systems Management (ISM), [online], v. 29, n.4, p. 258-268, 2012.

JANSSEN, M. et al. Transparency-by-design as a foundation for open government. Transforming Government: People, Process and Policy, v. 11, n. 1, p. 2-8, 2017.

KUČERA, J.; CHLAPEK, D.; NEČASKÝ, M. Open government data catalogs: Current approaches and quality perspective. In: International Conference on Electronic Government and the Information Systems Perspective. Springer Berlin Heidelberg, p. 152-166, 2013.

LÄMMERHIRT, D., RUBINSTEIN, M., MONTIEL, O. The State of Open Government Data in 2017: Creating meaningful open data through multi-stakeholder dialogue. Open Knowledge International. Report, 2017. 
LATHROP, D.; RUMA, L. Open Government: Collaboration, Transparency, and Participation in Practice; O’Reilly Media: Sebastopol, CA, USA, 2010.

LEE, G.; KWAK, Y. An open government maturity model for social media-based public engagement. Government Information Quarterly, v. 29, n. 4, p. 492-503, 2012.

LORENZO, S; CÉSAR, C. Open government: gobierno abierto. Jaén, España: Algón Editrores MMX, 2010.

LOURENÇO, R. P. An analysis of open government portals: A perspective of transparency for accountability. Government Information Quarterly, v. 32, n. 3, p. 323-332, 2015.

MACADAR, M. A.; KLEIN, R.; LUCIANO, E. Grau de Transparência de Dados Abertos Governamentais do site dados. rs. gov. br. Revista Economia \& Gestão, 2015.

MARISCAL, J. P. Del gobierno abierto a la transparencia proactiva: la experiencia del ifai en 2011. In: HOFMANN, A.; MURILLO, M. Evaluating the role of online data availability: The case of economic and institutional transparency in sixteen Latin American nations. International Political Science Review, v. 36, n. 1, p. 42-59, 2013.

MEIJER, A.; CURTIN, D.; HILLEBRANDT, M. Open government: connecting vision and voice. International Review of Administrative Sciences, v. 78, n. 1, p. 10-29, 2012.

OECD. Open Government in Latin America, OECD Public Governance Reviews, OECD Publishing, Paris, 2014. DOI https://doi.org/10.1787/9789264223639-en.

O'HARA, K. Data quality, government data and the open data infosphere. In AISB/IACAP World Congress 2012: Information Quality Symposium. Birmingham: The Society for the Study of Artificial Intelligence and Simulation of Behaviour. 2012.

OSZLAK, O. Gobierno abierto: promesas, supuestos, desafíos. In: Trabajo presentado en la VIII Conferencia Anual INPAE. 2012.

PISCOPO, A.; SIEBES, R.; HARDMAN, L. Predicting sense of community and participation by applying machine learning to open government data. Policy \& Internet, v. 9, n. 1, p. 55-75, 2017. 
POSSAMAI, A. J. Portal Brasileiro de Dados Abertos: novas práticas para o fortalecimento da democracia e da gestão pública na Era Digital. In: SILVA, Sivaldo Pereira da; BRAGATTO, Rachel Callai; SAMPAIO, Rafael Cardoso. (Org.). Democracia digital, comunicação política e redes: teoria e prática. 1ed.Rio de Janeiro: Letra \& Imagem, 2016, p. 365-392.

PYROZHENKO, V. Open government: Missing questions. Administration \& Society, v. 49, n. 10, p. 1494-1515, 2017.

RAMÍREZ-AlUJAS, A. V.; PEREZNIETO, J. A. B. (Org.). La promesa del gobierno abierto. México, DF: Instituto de Acceso a la Información Pública y Protección de Datos Personales del Distrito Federal, 2012.

ROBERTS, A. Blacked out: Government secrecy in the information age. Cambridge University Press, 2006.

SÁEZ MARTÍN, A.; DE ROSARIO, A.; PÉREZ, M. An international analysis of the quality of open government data portals. Social Science Computer Review, v. 34, n. 3, p. 298-311, 2016.

SCHROCK, A. Civic hacking as data activism and advocacy: A history from publicity to open government data. New Media \& Society, v. 18, n. 4, p. 581-599, 2016.

SCHROCK, A.; SHAFFER, G. Data ideologies of an interested public: A study of grassroots open government data intermediaries. Big Data \& Society, v. 4, n. 1, p. $2053951717690750,2017$.

VETRÒ, A. et al. Open data quality measurement framework: Definition and application to Open Government Data. Government Information Quarterly, v. 33, n. 2, p. 325-337, 2016.

WANG, H.; LO, J. Adoption of open government data among government agencies. Government Information Quarterly, v. 33, n. 1, p. 80-88, 2016.

WIJNHOVEN, F.; EHRENHARD, M.; KUHN, J. Open government objectives and participation motivations. Government Information Quarterly, v. 32, n. 1, p. 30-42, 2015.

WIRTZ, B.; BIRKMEYER, S. Open government: Origin, development, and conceptual perspectives. International Journal of Public Administration, v. 38, n. 5, p. 381-396, 2015. 
WIRTZ, B.; WEYERER, J.; RÖSCH, M. Citizen and open government: an empirical analysis of antecedents of open government data. International Journal of Public Administration, v. 41, n. 4 , p. $308-320,2017$.

YANG, T.; LO, J.; SHIANG, J. To open or not to open? Determinants of open government data. Journal of Information Science, v. 41, n. 5, p. 596-612, 2015.

YANG, T.; WU, Y. Examining the socio-technical determinants influencing government agencies' open data publication: A study in Taiwan. Government Information Quarterly, v. 33, n. 3, p. 378-392, 2016.

YU, H.; ROBINSON, D. G. The new ambiguity of 'open government'. UCLA Law Review Discourse, 2012.

ZUIDERWIJK, A. et al. Design principles for improving the process of publishing open data. Transforming Government: People, Process and Policy, v. 8, n. 2, p. 185-204, 2014.

ZUIDERWIJK, A.; JANSSEN, M.; DWIVEDI, Y. Acceptance and use predictors of open data technologies: Drawing upon the unified theory of acceptance and use of technology. Government Information Quarterly, v. 32, n. 4, p. 429-440, 2015.

${ }^{1}$ O presente trabalho foi realizado com apoio da Coordenação de Aperfeiçoamento de Pessoal de Nível Superior - Brasil (CAPES) - Código de Financiamento 001 - e com o apoio do Conselho Nacional de Desenvolvimento Científico e Tecnológico (CNPq).

2 Mestre em Comunicação e Cultura Contemporâneas pela Universidade Federal da Bahia (UFBA) na linha de comunicação política. Pesquisadora do Instituto Nacional de Ciência e Tecnologia em Democracia Digital (INCT.DD). Salvador - BA, Brasil. E-mail: mariadominguezcp@gmail.com.

${ }^{3}$ Doutora e mestre em Comunicação e Cultura Contemporâneas pela Universidade Federal da Bahia (UFBA) na linha de comunicação política. Graduada em Comunicação Social (Jornalismo) pela mesma instituição. Realizou estágio-doutoral na School of Public Affairs and Administration, Rutgers University (EUA) sob co-orientação da professora doutora Suzanne Piotrowski. Pesquisadora pós-doc (bolsista CAPES) do Instituto Nacional de Ciência e Tecnologia em Democracia Digital (INCT-DD). Membro dos grupos de pesquisa Comunicação, Internet e Democracia (UFBA); Pesquisa em Comunicação, Internet e Política (PUC-Rio) e Grupo de Pesquisa em Comunicação, Política e Tecnologia (UFPR). Salvador - BA, Brasil. E-mail: mariapaula.almada@gmail.com. 